SOUZA AG; AMARANTE CVT; DESCHAMPS FC; ERNANI PR. 2006. Calagem e adubação fosfatada promovem crescimento inicial e produção de hipericina em erva-de-São-João. Horticultura Brasileira 24: 421-425.

\title{
Calagem e adubação fosfatada promovem crescimento inicial e produ- ção de hipericina em erva-de-São-João
}

\author{
Alexandra Goede de Souza ${ }^{1 ; 2}$; Cassandro Vidal Talamini do Amarante ${ }^{1 ; 3}$; Francisco Carlos Deschamps ${ }^{4}$; \\ Paulo Roberto Ernani ${ }^{1 ; 3}$ \\ ${ }^{1}$ UDESC-CAV, C. Postal 281, 88502-970 Lages-SC; ${ }^{2}$ Aluna do Curso de Mestrado em Ciência do Solo, Bolsista da CAPES; ${ }^{3}$ Bolsista do \\ CNPq; ${ }^{4}$ EPAGRI, C. Postal 277, 88301-970 Itajaí-SC; E-mail: amarante@ cav.udesc.br
}

\section{RESUMO}

A erva-de-São-João é uma planta medicinal empregada no tratamento antidepressivo. A hipericina é considerada um dos compostos que contribui para o efeito medicinal da planta. Uma vez que a concentração e a quantidade do princípio ativo pode ser afetada pela nutrição das plantas, este trabalho teve por objetivo avaliar os efeitos do $\mathrm{pH}$ do solo e da adubação fosfatada sobre o crescimento inicial e a produção de hipericina em erva-de-São-João. O experimento foi realizado em Lages, SC, de julho a dezembro de 2003, em casa de vegetação. Utilizou-se delineamento experimental inteiramente casualizado (fatorial $4 \times 3$ ), correspondendo a quatro valores de $\mathrm{pH}(4,1 ; 5,5 ; 6,0$ e 6,5$)$ e três doses de $\mathrm{P}\left(0,50\right.$ e $100 \mathrm{mg} \mathrm{kg}^{-1} \mathrm{de}$ solo), com quatro repetições. Foram cultivadas duas plantas por vaso, em um Cambissolo Húmico Álico. Avaliou-se a produção de massa seca, a altura e o número de ramificações da parte aérea, o número de glândulas escuras nas folhas e a concentração e a quantidade total de hipericina na parte aérea. A produção de massa seca da parte aérea aumentou com a adição de $\mathrm{P}$ e, em maior magnitude, com a calagem. A altura das plantas somente foi influenciada pela calagem. O número de ramificações e de glândulas escuras e a concentração de hipericina aumentaram com a aplicação de $\mathrm{P}$ apenas na ausência de calagem, e com a calagem na ausência de P. Os maiores conteúdos de hipericina por vaso foram verificados nos tratamentos com pH 6,0 e 6,5 e doses de $\mathrm{P}$ de 50 e $100 \mathrm{mg} \mathrm{kg}^{-1}$ de solo.

Palavras-chave: Hypericum perforatum L., planta medicinal, fitoterápico, nutrição.

\begin{abstract}
Liming and phosphate fertilization promote initial growth and hipericin production in St. John's wort

St. Jonh's wort is a medicinal plant used for anti-depressive treatment. Hypericin is considered one of the compounds that contribute to the medicinal effect of the plant. Since the concentration and the total quantity of active ingredients may be affected by plant nutrition, this work was conducted to investigate the effects of soil $\mathrm{pH}$ and phosphorus (P) addition to the soil on initial growth and hypericin production of St. John's wort. The experiment was carried out in Lages, SC, from July until December of 2003, in a greenhouse. It followed a completely randomized factorial design (4 x 3), with four levels of $\mathrm{pH}(4.1,5.5,6.0$, and 6.5) and three doses of $\mathrm{P}(0,50$, and $100 \mathrm{mg} \mathrm{kg}^{-1}$ of soil), and four replicates. Two plants were cultivated per pot, under a Humic Inceptisol. Plants were harvested and assessed for aerial dry matter production, height, number of lateral shoots, number of dark glands in the leaves, aerial hypericin content and aerial total hypericin production. The aerial dry matter production increased with $\mathrm{P}$ addition and, in a larger amount, with liming. The plant height was only affected by the liming. The number of lateral shoots and dark glands in the leaves as well as the hipericin concentration only increased with $\mathrm{P}$ application in the soil without liming, and with liming in the soil that did not receive $\mathrm{P}$. The highest total hypericin production per pot was achieved in the treatments with $\mathrm{pH}$ values of 6.0 and 6.5 and $\mathrm{P}$ doses of 50 and $100 \mathrm{mg} \mathrm{kg}^{-1}$ of soil.
\end{abstract}

Keywords: Hypericum perforatum L., medicinal plants, phytoterapic, nutrition.

(Recebido para publicação em 13 de maio de 2005; aceito em 18 de dezembro de 2006)

\begin{abstract}
A erva-de-São-João (Hypericum perforatum L.) é uma planta herbácea, perene, pertencente à Família Hypericaceae, originária da Europa, Ásia e Norte da África, que se adapta bem em outras regiões temperadas. Esta planta possui propriedades anti-inflamatória, antiviral e antidepressiva reconhecidas desde a antiguidade (Kitanov, 2001). Atualmente, o extrato de erva-de-São-João está sendo usado principalmente no tratamento de depressões com graus leves a moderados, problema que afeta de 2 a $5 \%$ da população mundial (Poutaraud et al., 2001b).
\end{abstract}

O extrato de erva-de-São-João contém um grande número de constituintes químicos com propriedades farmacológicas confirmadas, como os flavonóides, ácidos fenólicos, taninos e hipericinas (iso, proto e pseudo hipericinas) (Nahrstedt \& Butterweck, 1997, Schwob et al., 2002; Briskin et al., 2000; Curtis \& Lersten, 1990). A hipericina é um metabólito secundário da planta que possui efeito antidepressivo comprovado, utilizada na forma de soluções, comprimidos, cápsulas, tabletes e xaropes (Di Carlo et al., 2001). A hipericina atua na inibição da enzima monoamino oxidase (MAO), responsável pela degradação de neurotransmissores (Butterweck et al.,
1998; Muller, 1997).

A erva-de-São-João possui dois tipos de glândulas: as translúcidas, que se distribuem uniformemente em toda a lâmina foliar, e as escuras, que estão localizadas preferencialmente na região marginal das folhas e das pétalas. Além das diferenças na aparência e distribuição, as glândulas diferem entre si quanto à composição interna (Curtis \& Lersten, 1990). As glândulas translúcidas consistem em cavidades sub-epidérmicas, delimitadas por duas finas camadas de células, como se estivessem abertas, enquanto que as escuras formam uma espécie de reservató- 
rio (Curtis \& Lersten, 1990; Fornasiero et al., 1998; Ciccarelli et al., 2001). Dentro do gênero Hypericum, algumas espécies apresentam somente as glândulas translúcidas, enquanto outras possuem ambas (Curtis \& Lersten, 1990). A hipericina é uma substância típica do gênero Hipericum e está localizada no interior das glândulas escuras (Cellarova et al., 1992).

A qualidade do fitomedicamento produzido está diretamente relacionada com a concentração total de hipericina presente na planta. Segundo Southwell \& Bourke (2001), a concentração de hipericina é dependente da quantidade e do tamanho das glândulas escuras produzidas pela planta, o que é afetado por condições ambientais e da própria planta. Os autores observaram que o teor de hipericina depende da variedade e da estação do ano. Quando foram produzidas no inverno, tanto as variedades de folhas largas como as de folhas estreitas apresentaram teores de hipericina de aproximadamente $100 \mathrm{mg} \mathrm{g}^{-1}$ de massa seca. No entanto, no verão, as variedades de folhas estreitas apresentaram teores superiores $\left(5.000 \mathrm{mg} \mathrm{g}^{-1}\right.$ de massa seca) em relação às variedades de folhas largas (3.000 $\mathrm{mg} \mathrm{g}^{-1}$ de massa seca). Segundo Poutaraud et al. (2001b), os teores de hipericina também variam entre as diferentes partes da planta, sendo maiores nos botões florais, seguidos das flores, folhas e ramos. A intensidade luminosa à qual a planta é submetida também altera o teor de hipericina. $\mathrm{O}$ aumento da intensidade luminosa (no comprimento de onda de $515 \mathrm{~nm}$ ) promove a transformação da proto-hipericina em hipericina, elevando o teor do fitoterápico ativo na planta (Briskin \& Gawienowski, 2001; Poutaraud et al., 2001a).

$\mathrm{O}$ cultivo in vitro de erva-de-SãoJoão é uma maneira alternativa para a obtenção do principio ativo vegetal de forma contínua, sem variação no conteúdo e qualidade da hipericina, por não estar submetido às oscilações climáticas e de solo (Santarém \& Astarita, 2003). O crescimento vegetativo e a produção de hipericina podem ser potencializados quando culturas celulares de $H$. perforatum são mantidas na ausência de luz e tratadas com ácido jasmônico (Walker et al., 2002).
A produção de hipericina também é influenciada pela nutrição das plantas. Baixos níveis de $\mathrm{N}$ no solo levam ao aumento da concentração de hipericina em erva-de-São-João (Briskin et al., 2000; Denke et al., 1999). O aumento na disponibilidade de $\mathrm{N}$ proporciona melhores condições de desenvolvimento para a planta, aumentando seu crescimento vegetativo, diluindo, porém, o teor de hipericina na parte aérea (Briskin et al., 2000). A adição de $\mathrm{Ca}, \mathrm{Mg}, \mathrm{K}$ e $\mathrm{P}$ causou efeito similar ao $\mathrm{N}$ sobre o crescimento vegetativo de $H$. perforatum, Urtica dióica, Hyssopus officinalis, Achillea millefolium e Calendulas officinalis, demonstrando o efeito benéfico dos principais macronutrientes para o desenvolvimento vegetativo das plantas (Lieres et al., 1994).

A elevada acidez e a baixa de disponibilidade de $\mathrm{P}$ presentes na maioria dos solos brasileiros representam grandes restrições ao desenvolvimento das plantas cultivadas (Ernani \& Almeida, 1986; Ernani et al., 1996 e 2000). No caso do cultivo comercial de erva-de-São-João, estes fatores podem ter impactos substanciais no crescimento das plantas e na produção de hipericina.

O presente estudo objetivou avaliar o efeito da calagem e da adubação fosfatada na produção de hipericina em erva-de-São-João.

\section{MATERIAL E MÉTODOS}

O experimento foi conduzido em casa de vegetação, no Centro de Ciências Agroveterinárias (CAV), em Lages, SC, de julho a dezembro de 2003. As mudas de Hypericum perforatum L. foram obtidas por meio de propagação assexuada, de material proveniente da Estação Experimental de Itajaí, da Empresa de Pesquisa Agropecuária e Extensão Rural de Santa Catarina S.A. (Epagri).

Adotou-se o delineamento experimental inteiramente casualizado, seguindo um fatorial $4 \times 3$, correspondendo a quatro valores de $\mathrm{pH}$ $(4,1 ; 5,5 ; 6,0$ e 6,5$)$ e três doses de fósforo (0, 50 e $100 \mathrm{mg} \mathrm{kg}^{-1}$ de solo), com quatro repetições. Cada unidade experimental foi constituída por um vaso contendo $1,0 \mathrm{~kg}$ de solo (base seca) e duas plantas. Foi utilizado um CAMBISSOLO HÚMICO ÁLICO (Empara, 1999), coletado em área de campo nativo, na camada superficial de 0 a $20 \mathrm{~cm}$ de profundidade. O solo apresentava pH em água 4,1 e SMP 4,3, Al trocável $=7,3 \mathrm{cmol}_{\mathrm{cg}} \mathrm{kg}^{-1}$, matéria orgânica $=43 \mathrm{~g} \mathrm{~kg}^{-1}$, argila $=520 \mathrm{~g} \mathrm{~kg}^{-1}$, areia $=290 \mathrm{~g} \mathrm{~kg}^{-1}$, silte $=360 \mathrm{~g} \mathrm{~kg}^{-1}, \mathrm{P}$ e $\mathrm{K}($ Mehlich-1)=4,85 e 144,0 mg kg-1, respectivamente, e $\mathrm{Ca}$ e $\mathrm{Mg}=1,0$ e 0,6 $\mathrm{cmol}_{\mathrm{c}} \mathrm{kg}^{-1}$, respectivamente.

Os níveis de $\mathrm{pH}$ utilizados neste trabalho foram definidos considerando o $\mathrm{pH}$ do solo não corrigido $(4,1)$ e os valores de $\mathrm{pH}$ recomendados em cultivos agrícolas pela Comissão de Fertilidade do Solo - RS/SC (2004) para os estados de Santa Catarina e Rio Grande do Sul, correspondentes a 5,5, 6,0 ou 6,5. Para elevação do $\mathrm{pH}$ utilizou-se calcário dolomítico finamente peneirado, preparado em laboratório (com PRNT de $100 \%$, e conteúdo de $\mathrm{CaCO}_{3}$ e $\mathrm{MgCO}_{3}$ na proporção de $3: 1$, em peso, com $31 \%$ de $\mathrm{Ca}$ e $6 \%$ de $\mathrm{Mg}$ ), nas doses de 7,5, 10,5 e $14,5 \mathrm{~g} \mathrm{~kg}^{-1}$. O calcário foi aplicado dois meses antes do plantio das mudas, visando permitir a correção do $\mathrm{pH}$ deste solo para os valores desejados, conforme descrito por Ernani \& Almeida (1986) e Ernani et al. (1996 e 2000). Para suprir as doses de 0,50 e $100 \mathrm{mg} \mathrm{kg}^{-1}$ de $\mathrm{P}$, foi utilizado fosfato de potássio monobásico $\left(\mathrm{KH}_{2} \mathrm{PO}_{4}\right)$. Para evitar um possível efeito do $\mathrm{K}$ adicionado juntamente por esta fonte, o mesmo foi uniformizado em todos os tratamentos por meio da adição suplementar com sulfato de potássio. O nitrogênio foi aplicado em todos os tratamentos na dose de $100 \mathrm{mg} \mathrm{kg}^{-1}$ de solo, na forma de uréia, antes do plantio das mudas.

No mês de novembro, foram realizadas três aplicações foliares de boro e zinco, na concentração de $0,1 \%$ de sulfato de zinco e $0,1 \%$ de bórax, em todos os tratamentos, para garantir o suprimento adequado destes nutrientes.

Cinco meses após o plantio das mudas, foram determinados o número de ramificações, a altura das plantas e o número de glândulas escuras nas folhas. A determinação do número de glândulas foi feita por contagem, com auxílio 
de uma lupa, em quatro folhas totalmente expandidas por planta, no quinto e sexto pares de folhas a partir do ápice. Posteriormente, as plantas foram colhidas para a avaliação da produção de massa seca e do conteúdo de hipericina na parte aérea. A massa seca foi determinada após secagem da parte aérea das plantas a $65^{\circ} \mathrm{C}$ durante $48 \mathrm{~h}$, em estufa com circulação forçada de ar.

A determinação do conteúdo de hipericina na parte aérea foi realizada na Estação Experimental da Epagri, em Itajaí, SC. Após secagem e trituração do material vegetal, aproximadamente $300 \mathrm{mg}$ de cada amostra foram lavadas por Soxhlet com dietil éter durante 16 horas, para remoção da clorofila e outros pigmentos. Após a evaporação do solvente, a hipericina foi extraída das amostras usando-se $3 \mathrm{~mL}$ de etanol absoluto, durante 24 horas, segundo metodologia descrita por Southwell \& Bourke (2001). Em seguida, o material foi filtrado, o extrato etanólico das amostras foi diluído com etanol absoluto, e a hipericina foi quantificada por meio de espectrofotometria, no comprimento de onda $(\lambda)$ de $589 \mathrm{~nm}$. Como a clorofila não foi completamente removida das amostras, sua leitura, feita no $\lambda$ de $660 \mathrm{~nm}$, foi subtraída da leitura da hipericina.

Para uma análise qualitativa da presença da hipericina, as amostras foram submetidas à cromatografia em camada delgada (CCD) de sílica-gel G-60 (Merck). O etanol foi removido das amostras por evaporação, e a fase sólida re-suspensa em 50L de etanol absoluto. Alíquotas desse substrato foram analisadas por CCD, com a fase móvel composta de tolueno-etanol-metanol (30:50:20, v/v/v), seguindo uma metodologia modificada descrita por Kitanov (2001). A presença da hipericina foi observada em luz UV no $\lambda$ de $366 \mathrm{~nm}$, antes da evaporação da fase móvel. Os perfis cromatográficos das amostras foram comparados com o perfil da amostra padrão (Briskin \& Gawienowski, 2001).

Os resultados foram analisados estatisticamente com o programa SAS (SAS Institute, Inc.) e as médias comparadas pelo teste de Duncan $(P<0,05)$.

\section{RESULTADOS E DISCUSSÃO}

A calagem apresentou maior efeito promotor do crescimento das plantas do

Tabela 1. Produção de massa seca da parte aérea $\left(\mathrm{g} \mathrm{vaso}^{-1}\right)$ de erva-de-São-João em função do $\mathrm{pH}$ e de doses de P aplicadas ao solo. Lages- SC, UDESC, 2003.

\begin{tabular}{lcccc}
\hline \multirow{2}{*}{$\mathbf{l}$} & $\mathbf{5}$ & \multicolumn{4}{c}{ Doses de $\mathbf{P}\left(\mathbf{m g ~ k g}^{-1}\right)$} \\
\cline { 2 - 5 } & $0,75 \mathrm{Bb}^{1}$ & $\mathbf{5 0}$ & $\mathbf{1 0 0}$ & Média \\
\hline 4,1 & $2,58 \mathrm{Aa}$ & $3,07 \mathrm{Ab}$ & $1,36 \mathrm{Ad}$ & $1,18 \mathrm{c}$ \\
5,5 & $3,04 \mathrm{Aa}$ & $3,56 \mathrm{Aab}$ & $3,10 \mathrm{Ac}$ & $2,92 \mathrm{~b}$ \\
6,0 & $2,79 \mathrm{Ba}$ & $4,29 \mathrm{Aa}$ & $4,76 \mathrm{Aa}$ & $3,51 \mathrm{a}$ \\
6,5 & $2,29 \mathrm{~B}$ & $3,09 \mathrm{~A}$ & $3,29 \mathrm{~A}$ & $2,95 \mathrm{a}$ \\
\hline Média & 22,4 & 18,3 & 15,9 & 18,6 \\
\hline C.V. $(\%)$ & &
\end{tabular}

${ }^{1}$ Médias seguidas da mesma letra, minúscula nas colunas e maiúscula nas linhas, não diferem entre si pelo teste de Duncan $(P<0,05)$.

Tabela 2. Altura e número de ramificações na parte aérea da erva-de-São-João em função do $\mathrm{pH}$ e de doses de P aplicadas ao solo. Lages- SC, UDESC, 2003.

\begin{tabular}{lllll}
\hline \multirow{5}{*}{$\mathbf{p H}$} & $\mathbf{5}$ & \multicolumn{5}{c}{ Dose de $\mathbf{P}\left(\mathbf{m g ~ k g}^{-1}\right)$} \\
\hline \multicolumn{5}{c}{ Altura (cm) } \\
\cline { 2 - 5 } & \multicolumn{5}{c}{$\mathbf{1 0 0}$} & Média \\
\hline 4,1 & $25,7 \mathrm{Ab} 1$ & $29,3 \mathrm{Ab}$ & $23,4 \mathrm{Ab}$ & $26,2 \mathrm{~b}$ \\
5,5 & $35,0 \mathrm{Aa}$ & $36,4 \mathrm{Aa}$ & $33,9 \mathrm{Aa}$ & $35,1 \mathrm{a}$ \\
6,0 & $33,2 \mathrm{Aab}$ & $31,2 \mathrm{Aab}$ & $32,2 \mathrm{Aa}$ & $32,2 \mathrm{a}$ \\
6,5 & $36,0 \mathrm{Aa}$ & $34,7 \mathrm{Aab}$ & $33,7 \mathrm{Aa}$ & $34,8 \mathrm{a}$ \\
\hline Média & $32,5 \mathrm{~A}$ & $32,9 \mathrm{~A}$ & $30,8 \mathrm{~A}$ & 32,1 \\
\hline C.V. (\%) & 17,5 & 10,2 & 17,4 & 15,1 \\
\hline \multicolumn{5}{c}{$\mathbf{N}^{\circ}$ de ramificações/planta } \\
\hline 4,1 & $7,5 \mathrm{Bb}$ & $26,7 \mathrm{Aa}$ & $25,0 \mathrm{Aa}$ \\
5,5 & $28,5 \mathrm{Aa}$ & $29,2 \mathrm{Aa}$ & $30,9 \mathrm{Aa}$ & $29,5 \mathrm{a}$ \\
6,0 & $28,0 \mathrm{Aa}$ & $24,0 \mathrm{Aa}$ & $23,1 \mathrm{Aa}$ & $25,0 \mathrm{a}$ \\
6,5 & $25,6 \mathrm{Aa}$ & $29,2 \mathrm{Aa}$ & $26,5 \mathrm{Aa}$ & $27,1 \mathrm{a}$ \\
\hline Média & $22,4 \mathrm{~B}$ & $27,3 \mathrm{~A}$ & $26,4 \mathrm{AB}$ & 25,4 \\
\hline C.V. (\%) & 31,9 & 17,6 & 20,3 & 23,1 \\
\hline
\end{tabular}

${ }^{1}$ Médias seguidas da mesma letra, minúscula nas colunas e maiúscula nas linhas, não diferem entre si pelo teste de Duncan $(P<0,05)$.

que a adubação fosfatada (Tabela 1). A elevação do $\mathrm{pH}$ do solo para 5,5 aumentou o rendimento de massa seca da parte aérea em aproximadamente $244 \%$ em relação ao tratamento sem calagem, na ausência da fertilização fosfatada. A aplicação de $50-100 \mathrm{mg} \mathrm{kg}^{-1}$ de $\mathrm{P}$, por outro lado, aumentou o rendimento de massa seca em aproximadamente $86 \%$ em relação ao tratamento sem adição de $\mathrm{P}$, na ausência de calcário.

A adubação fosfatada somente aumentou a massa seca da parte aérea no tratamento sem calagem e naquele com pH 6,5 (Tabela 1).

Em valores baixos de $\mathrm{pH}$, parte do $\mathrm{P}$ presente no solo permanece fortemente retido nos sítios de ligação da fase sólida, e também na forma de precipitados de fosfato de ferro e de alumínio, permanecendo pouco disponível às plantas
(Ernani et al., 2000). Com a elevação do $\mathrm{pH}$, aumentam as cargas negativas do solo, diminui a solubilidade do ferro e do alumínio, e, com isso aumenta a disponibilidade de $\mathrm{P}$ na solução do solo (Ernani et al., 2000), não havendo, portanto, o efeito da sua aplicação, uma vez que o fornecido naturalmente pelo solo (com a elevação do $\mathrm{pH}$ ) é suficiente para aumentar a produção de massa seca da parte aérea das plantas (Tabela 1). Com a correção do $\mathrm{pH}$ para 6,5 , há novamente uma redução no teor de $\mathrm{P}$ na solução do solo, devido à adição de calcário, que gera um grande aumento na quantidade de Ca no solo, favorecendo a precipitação do $\mathrm{P}$ na forma de fosfato de cálcio (Akinremi \& Cho, 1991). Portanto, em pH 6,5 observa-se um aumento na produção de massa seca na parte aérea das planta de erva-de-São-João com a adi- 
ção de P (Tabela 1). No entanto, a maior produção de massa seca da parte aérea $\left(4,76 \mathrm{~g} \mathrm{vaso}^{-1}\right)$ foi obtida no tratamento com pH 6,5 e na dose de $100 \mathrm{mg} \mathrm{kg}^{-1}$ de $\mathrm{P}$, mostrando o efeito benéfico da elevação do $\mathrm{pH}$ do solo e da aplicação de $\mathrm{P}$ para o desenvolvimento da erva-de São-João.

A altura das plantas somente foi afetada pela calagem, sendo, na média dos valores, 29,9\% maior em relação ao tratamento sem calcário. No entanto, não houve diferença significativa entre os tratamentos que receberam calcário, independente da dose de $\mathrm{P}$, sugerindo que a correção do $\mathrm{pH}$ para 5,5 seria suficiente para não restringir a altura das plantas (Tabela 2). O número de ramificações também aumentou com a calagem, porém somente no solo que não recebeu $\mathrm{P}$, sendo igual para os três níveis de calagem. A aplicação de $\mathrm{P}$ promoveu maior ramificação das plantas somente na ausência de calcário, entretanto em menor magnitude relativa ao efeito da calagem (Tabela 2).

A elevação do $\mathrm{pH}$ do solo aumentou o número de glândulas escuras nas folhas, independente da dose aplicada de P. A adição de P, no entanto, aumentou o número de glândulas escuras somente na ausência de calcário (Tabela 3).

Os extratos da erva-de-São-João analisados em cromatografia de camada delgada (CCD), apresentaram bandas rosadas na placa de sílica gel, caracterizando a presença da hipericina (dados não apresentados). A identificação das bandas de hipericina foi baseada na comparação com a banda da amostra padrão, através de separação e fluorescência em luz U.V. no $\lambda$ de $366 n m$ e dos espectros de absorção (dados não apresentados). A leitura do pico máximo de absorbância da hipericina no padrão ocorreu no $\lambda$ de $589 \mathrm{~nm}$, valor muito similar ao observado por outros autores (Southwell \& Bourke, 2001; Kitanov, 2001), comprovando a presença de hipericina nos extratos.

A concentração de hipericina aumentou com a elevação do $\mathrm{pH}$ do solo para valores iguais ou superiores a 5,5, independente da aplicação de P (Tabela 3). A adição de $\mathrm{P}$ aumentou a concentração de hipericina nas folhas, porém somente na ausência de calcário (Tabela 3 ). Observou-se também que na ausência de calagem, a planta necessitou de doses maiores de $\mathrm{P}$ para aumentar a síntese e o acúmulo de hipericina do que para a for-

Tabela 3. Número de glândulas escuras por folha, concentração ( $\mathrm{mg} \mathrm{g}^{-1}$ de massa seca) e conteúdo de hipericina $\left(\mathrm{mg}\right.$ vaso $\left.^{-1}\right)$ da erva-de-São-João em função do $\mathrm{pH}$ e de doses de $\mathrm{P}$ aplicadas ao solo. Lages- SC, UDESC, 2003.

\begin{tabular}{|c|c|c|c|c|}
\hline \multirow{2}{*}{ pH } & \multicolumn{4}{|c|}{ Dose de $\mathrm{P}\left(\mathrm{mg} \mathrm{kg}^{-1}\right)$} \\
\hline & 0 & 50 & 100 & Média \\
\hline \multicolumn{5}{|c|}{ Glândulas escuras/folha } \\
\hline$\overline{4,1}$ & $4,5 \mathrm{Bc} 1$ & $8,9 \mathrm{Aa}$ & $8,9 \mathrm{Aa}$ & $7,4 \mathrm{c}$ \\
\hline 5,5 & 11,7 Aab & $8,1 \mathrm{Aa}$ & $9,1 \mathrm{Aa}$ & $9,7 a b$ \\
\hline 6,0 & $8,5 \mathrm{Ab}$ & $10,1 \mathrm{Aa}$ & $7,2 \mathrm{Aa}$ & $8,6 \mathrm{bc}$ \\
\hline 6,5 & $12,5 \mathrm{Aa}$ & $11,0 \mathrm{Aa}$ & $10,7 \mathrm{Aa}$ & $11,4 \mathrm{a}$ \\
\hline Média & $9,3 \mathrm{~A}$ & $9,5 \mathrm{~A}$ & $9,0 \mathrm{~A}$ & 9,3 \\
\hline C.V. $(\%)$ & 23,2 & 25,3 & 29,4 & 26,0 \\
\hline \multicolumn{5}{|c|}{ Concentração de hipericina ( $\mathrm{g} \mathrm{g}^{-1}$ de massa seca) } \\
\hline 4,1 & $428,4 \mathrm{Bb}$ & $464,8 \mathrm{Ba}$ & $687,1 \mathrm{Aa}$ & $526,8 \mathrm{~b}$ \\
\hline 5,5 & 644,6 Aab & $652,7 \mathrm{Aa}$ & $678,6 \mathrm{Aa}$ & 658,6 a \\
\hline 6,0 & $695,3 \mathrm{Aa}$ & $679,3 \mathrm{Aa}$ & $670,1 \mathrm{Aa}$ & 681,6 a \\
\hline 6,5 & $643,1 \mathrm{Aab}$ & $658,8 \mathrm{Aa}$ & $673,0 \mathrm{Aa}$ & 658,3 a \\
\hline Média & $602,9 \mathrm{~A}$ & $613,9 \mathrm{~A}$ & $677,2 \mathrm{~A}$ & 631,3 \\
\hline C.V. $(\%)$ & 24,7 & 22,0 & 20,5 & 22,4 \\
\hline \multicolumn{5}{|c|}{ Conteúdo de hipericina $(\mathrm{g}$ vaso-1) } \\
\hline 4,1 & $316,1 \mathrm{Cb}$ & $656,3 \mathrm{Bb}$ & $934,0 \mathrm{Ac}$ & $635,4 \mathrm{c}$ \\
\hline 5,5 & $1679,2 \mathrm{Aa}$ & $1968,1 \mathrm{Aa}$ & $2084,1 \mathrm{Ab}$ & $1910,5 \mathrm{~b}$ \\
\hline 6,0 & $1966,7 \mathrm{Aa}$ & $2433,4 \mathrm{Aa}$ & $2629,1 \mathrm{Aab}$ & 2343,0 a \\
\hline 6,5 & $1804,2 \mathrm{Ba}$ & $2823,5 \mathrm{ABa}$ & $3184,4 \mathrm{Aa}$ & $2604,0 \mathrm{a}$ \\
\hline Média & $1441,5 \mathrm{~B}$ & $1970,3 \mathrm{~A}$ & $2207,9 \mathrm{~A}$ & 1873,2 \\
\hline C.V. (\%) & 23,9 & 27,1 & 25,9 & 26,4 \\
\hline
\end{tabular}

${ }^{1}$ Médias seguidas da mesma letra, minúscula nas colunas e maiúscula nas linhas, não diferem entre si pelo teste de Duncan $(P<0,05)$.

mação das glândulas escuras (Tabela 3). Resultados similares também foram relatados por Formasiero et al. (1998) e Briskin \& Gawienowski (2001).

O conteúdo de hipericina por vaso acompanhou a produção de massa seca, sendo mais alto nos tratamentos com $\mathrm{pH}$ igual ou superior a 6,0 e que receberam as doses de $\mathrm{P}$ a partir de $50 \mathrm{mg} \mathrm{kg}^{-1}$ (Tabelas 1 e 3 ).

Desta maneira, a correção do pH e a adição de $\mathrm{P}$ ao solo permitem aumentar as produções de massa vegetativa sem redução no teor do princípio ativo na parte aérea em erva-de-São-João. Resultados similares foram relatados por Azizi \& Omidbaigi (2002) aplicando N e P ao solo. O N, em doses elevadas, promove aumento da massa seca e redução da concentração de hipericina por diluição (Briskin et al., 2000). No entanto, a adição combinada de $\mathrm{N}$ e $\mathrm{P}$ ao solo aumenta a produção total de hipericina nas plantas (Azizi \& Omidbaigi, 2002).

Os resultados obtidos mostram que para o cultivo da erva-de-São-João, a calagem combinada com a adubação fosfatada promove o rendimento de massa seca e de hipericina. Quando considerados os efeitos individuais desses fatores, a diminuição da acidez do solo teve maior contribuição sobre a resposta desta planta em relação a adição de $P$. O cultivo de plantas medicinais normalmente segue preceitos de produção orgânica ou agroecológica. Dessa forma, apenas a correção do $\mathrm{pH}$ para 6,0-6,5, sem a adição de fontes prontamente solúveis de $\mathrm{P}$, viabiliza o cultivo orgânico de erva-de-São-João em solos ácidos, visando a produção de hipericina. Todavia, em sistema convencional, visando atingir altos rendimentos de hipericina, recomenda-se a aplicação adicional de 50-100 $\mathrm{mg} \mathrm{kg}^{-1}$ de $\mathrm{P}$.

\section{AGRADECIMENTOS}

Os autores agradecem ao Conselho Nacional de Desenvolvimento Científico e Tecnológico (CNPq) e a Coordenação de Aperfeiçoamento de Pessoal de Nível Superior (CAPES) pelo apoio financeiro a este projeto. 


\section{REFERÊNCIAS}

AKINREMI OO; CHO CM. 1991. Phosphate transport in calcium-saturated systems: II Experimental results in a model system. Soil Science Society of American Journal 55: 1282 1287.

AZIZI M; OMIDBAIGI R. 2002. Effect of NP supply on herb yield, hypericin content and cadmium accumulation of St. John's Wort (Hypericum perforatum L.). Acta Horticulturae 576: 267-271.

BRISKIN DP; GAWIENOWSKI MC. 2001. Differential effects of light and nitrogen on production of hypericins and leaf glands in Hypericum perforatum. Plant Physiology and Biochemistry 39: 1075-1081.

BRISKIN DP; LEROY A; GAWIENOWSKI MC. 2000. Influence of nitrogen on the production of hypericins by St. John's Wort. Plant Physiology and Biochemistry 38: 413-420.

BUTTERWECK V; PETEREIT F; WINTERHOFF H; NAHRSTEDT A. 1998. Solubilized hypericin and psedohypericin from Hypericum perforatum exert antidepressant activity in the forced swimming test. Planta Medica 64: 291-294.

CELLAROVA E; KIMAKOVA GM BRUTOVSKA R. 1992. Multiple shoot formation and phenotypic changes of regenerants in Hypericum perforatum L. Acta Biotechnologica 12: 45-52.

CICCARELLI D; ANDREUCCI AC; PAGNI AM. 2001. Translucent glands and secretory canals in Hypericum perforatum L. (Hypericaceae): Morphological, anatomical and histochemical studies during the course of Ontogenesis. Annals of Botany 88: 637-644.

COMISSÃO DE FERTILIDADE DO SOLO-RS/ $\mathrm{SC}$ (CFS-RS/SC). 2004. Recomendações de adubação e de calagem para os Estados do Rio Grande do Sul e de Santa Catarina. 3. ed. Passo Fundo: SBCS-Núcleo Regional Sul. $394 \mathrm{p}$.
CURTIS JD; LERSTEN NR. 1990. Internal secretory structure in Hypericum: $H$. perforatum L. and $H$. balearicum L. New Phytologist 114: 571-580.

DENKE A; SCHEMPP H; MANN E; SCHNEIDER W; ELSTERN EF. 1999. Biochemical activities of extracts from Hypericum perforatum L. 4th communication: influence of different cultivation methods. Arzneimittel-Forschung 49: 120-125.

DI CARLO G; BORRELLI F; ERNST E; IZZO AA. 2001. St John's wort: Prozac from the plant kingdom. Pharmacological Sciences 22: 292-297.

ERNANI PR; NASCIMENTO JAL; CAMPOS ML; CAMILLO RJ. 2000. Influência da combinação de fósforo e calcário no rendimento de milho. Revista Brasileira de Ciência do Solo 24: 537-544.

ERNANI PR; FIGUEIREDO ORA; BECEGATO V; ALMEIDA JA. 1996. Decréscimo da retenção de fósforo no solo pelo aumento do $\mathrm{pH}$ Revista Brasileira de Ciência do Solo 20: 159162

ERNANI PR; ALMEIDA LA. 1986. Comparação de métodos analíticos para avaliar a necessidade de calcário dos solos de estado de Santa Catarina. Revista Brasileira de Ciência do Solo 10: 143-150.

FORNASIERO RB; BIANCHI A; PINETTI A. 1998. Anatomical and ultrastructural observations in Hypericum perforatum L. leaves. Journal Herbs Spices Medical Plants 5: 21-33.

KITANOV GM. Hypericin and pseudohypericin in some Hypericum species. 2001. Biochemical Systematics and Ecology 29: 171178.

LIERES AL von; VOLKMANN B; LIERES AL von. 1994. Relationship between fertilizer, nutrient withdrawal, and composition of different medicinal plants in pot experiment. In: CONGRESS OF ALTERNATIVES IN LAND USE AND THE PRODUCTION AND UTILIZATION OF AGRICULTURAL PRODUCTS, 19. Annals...Germany: VDLUFA. p. 541-544.
MULLER WE. 1997. Effect of Hypericum extract (LI 160) in biochemical models of antidepressant activity. Pharmacopsychiatry 30: 113-116.

NAHRSTEDT A; BUTTERWECK V. 1997. Biologically active and other chemical constituents of the herb Hypericum perforatum L. Pharmacopsychiatry 30: 129-134.

POUTARAUD A; GREGORIO F Di; TIN VCF; GIRARDIN P. 2001a. Effect of light on hypericins contents in fresh flowering top parts and in an extract of St. John's wort (Hypericum perforatum). Planta Medical 64: 254-259.

POUTARAUD A; LOBSTEIN A; GIRARDIN P; WENIGER B. 2001b. Improved procedure for the quality control of Hypericum perforatum L. Phytochemical Analysis 12: 355-362.

SANTARÉM ER; ASTARITA LV. 2003. Multiple shoot formation in Hypericum perforatum $\mathrm{L}$. and hypericin production. Brazilian Journal of Plant Physiology 15: 43-47.

SCHWOB I; BESSIRÈ JM; VIANO J. 2002. Composition of the essential oils of Hypericum perforatum L. from southeastern France. Comptes Rendus Biologies 325: 781-785.

SOUTHWELL IA; BOURKE CA. 2001. Seasonal variation in hypericin content of Hypericum perforatum. L. Biochemical Systematics and Ecology 56: 437-441.

WALKER TS; BAIS HP; VIVANCO JM. 2002. Jasmonic acid-induced hypericin production in cell suspension cultures of Hypericum perforatum L. (St. John's wort). Phytochemistry 60: 289-293. 